\title{
LE ROLE DU CAPITAL BANCAIRE DANS LES COLONIES PORTUGAISES DE L'ANGOLA ET DE ST.TOME DE 1864 AU DEBUT DU XXE SIECLE.
}

Adelino Torres

ISE, Universidade Técnica de Lisboa

La Banque National d'Outremer - en portugais: "Banco Nacional Ultramarino" ou BNU - fondée en 1864, entre dans la catégorie des Banques Coloniales qui sont apparues avec l'abolition officielle de l'esclavage. Ces banques ne se limitaient pas, comme l'a montré l'économiste portugais du début du siècle, Bento Carqueja, aux opérations ordinaires d'escompte et de dépôt. Elles étaient également des établissements de crédit agricole prêtant sur les récoltes futures, aussi bien que des banques d'émission et de circulation ${ }^{1}$. En général, dans les différents pays où elles furent créées, elles bénéficiaient de privilèges exceptionnels et avaient des attributions spéciales.

Les banques coloniales avaient donc la faculté d'émettre des billets, non pas sous la forme de liberté d'émission (banking principie) mais dans la modalité de "principe de circulation" (currency principie). En général on leur imposait deux obligations:

[1] La somme totale de la monnaie fiduciaire en billets ne pouvait excéder le triple des réserves métalliques (or);

[2] Le montant total des billets en circulation, des dépôts à vue et d'autres dettes de la banque, ne pouvait excéder le triple du capital social².

Les circonstances de la création de cette banque coloniale qu'a été la BNU (Banque Nationale d'Outremer), les privilèges dont elle a bénéficiés et les résultats de son activité, méritent quelque attention puisqu'ils constituent des éléments

\footnotetext{
1 Bento Carqueja, O Futuro de Portugal - Questões Económicas e Sociais (Lisbonne, 1900), 307.

2 Ibid., 308.
} 
d'une certaine façon indispensables pour la compréhension d'une problématique qui dépasse le cas spécifique de la BNU.

L'appréciation de l'action de cette banque en Angola et à St. Tomé n'est pas à proprement parler une tâche simple si l'on s'en tient uniquement aux Rapports Annuels de cette institution bancaire. Néanmoins, en lisant attentivement ces Rapports à partir de 1865 et en les comparant en même temps avec d'autres sources, il est possible de circonscrire les grandes lignes et les principaux résultats de son action en Angola et dans les lies de St. Tomé et Principe.

Sur les actionnaires de la BNU on sait peu de chose à vrai dire. Dans son Rapport Annuel de 1880 la banque fait référence à la question (plus tard, au début du XXe siècle, elle sera un peu plus explicite) et offre les données suivantes:

[a] 1014 actionnaires sont présentés nominalement (y compris 404 ayant moins de 10 actions) dont:

-200 femmes, veuves et orphelins -68 mineurs sous tutelle -16 hôpitaux, Miséricordes, Asiles et Institutions de Bienfaisance

[b] 9406 actionnaires possédant des titres au porteur

Total: 10420 actionnaires.

On ignore donc tout de ceux qui possèdent 90\% du capital. La BNU affirme dans son Rapport de 1890 que le capital de la banque représente: "les épargnes du travail obtenues par le labeur de plus d'une génération, le pécule du pauvre et finalement la dotation au soutien de la vieillesse, l'abri du malheur et de la souffrance, pécule confié à la garde d'une institution de crédit, laquelle a. aujourd'hui plus que jamais, l'absolu devoir de revendiquer et de défendre légalement ce dépôt sacré3.

En dépit de cette "argumentation" il y a tout lieu de croire que les grands porteurs d'actions appartiennent surtout à la grande bourgeoisie mercantile et agraire de Lisbonne et du centre du pays.

3 Banco Nacional Ultramarino, Relatórios do Banco Nacional Ultramarino desde o ano da sua fundação em 1865 ate 1889 (Lisbonne, 1890), 570. 
A l'origine de la fondation de la BNU on trouve les frères Chamiço. Francisco Oliveira Chamiço, fondateur et premier gouverneur de la Banque, fut gérant d'une grande firme commerciale (familiale), Secrétaire de I'Association Commerciale de Lisbonne et participa a la fondation de deux Compagnies d'Assurances. Son frère, Fortunato Oliveire Chamiço, avait fondé la Maison Fortunato Chamiço \& Compagnie qui se tr an former a plus tard dans la Banque Totta. II a également fait partie du premier Conseil d'Administration de la Compagnie des Chemins de Fer Portugais 4.

Sans vouloir faire l'historique de la BNU, il n'est cependant pas inutile de rappeler qu'il y avait des liens familiaux entre Chamiço et le Ministre de la Marine et I'Outremer, José da Silva Mendes Leal ${ }^{5}$. Comme il est évident, cela a donne lieu à des interprétations diverses que nous ne discuterons pas ici. Ce qui est certain c'est que l'action du Ministre a été "déterminante dans l'événement" de la fondation de la Banque Nationale d'Outremer. En dépit des critiques généralisées de la presse et de beaucoup de secteurs de l'opinion, il n'y a eu aucun concours public comme il aurait été normal: la Banque a été créée sur proposition du Gouvernement ${ }^{6}$. Les privilèges très importants dont elle bénéficiait pour une période de 15 ans, éventuellement renouvelable, étaient, selon l'article 5 de la Charte de Loi du 16 mai 1864:

- L'exclusif de fondation et d'administration d'institutions bancaires dans toutes les Provinces d'Outremer, sauf Macau;

- Une subvention de 30 "contos" annuels";

- Affranchissements de toutes contributions et impôts ;

- Possibilités d'effectuer dans les colonies "toutes les opérations propres aux Banques de circulation aussi bien que de crédit agricole". Etc.

\footnotetext{
4 Vd João de Sousa da Camara, Missão Pedagógica da Banca Nacional (Lisbonne, 1968) où un chapitre entier est con sacré aux frères Chamico.

5 Cf. Fernando Emydgio da Silva, O Banco Nacional Ultramarino ao tempo da sua formação (Lisbonne, 1964), 8.

${ }^{6}$ Pourquoi n'a-t-on pas ouvert concours public pour la création de la Banque? Réponse du Ministre Mendes Leal: "Parce que ce principe, excellent en soi, ne peut pas toujours s'appliquer. Dans ce cas ce serait très risqué: n'importe quelle proposition fantasque, aventureuse ou mal intentionnée pourrait tout compromettre, freinant politiquement la résolution urgente et correcte du problème". Cité in Silva, 20.

7 Un "conto" vallait un million de réis, Banco Nacional.
} 
Pratiquement la seule limitation imposée par l'Etat en échange des privilèges de la Banque a consiste en fixer le maximum de l'intérêt: 8\% pour les opérations de crédit immobilier et $12 \%$ pour toutes les autres opérations.

En plaçant la BNU dans la constellation sociale portugaise, on remarque tout de suite plusieurs inter-relations:

- Avec la Haute Administration (Chamiço/Mendes Leal, Andrade Corvo et Neves Ferreira8;

- avec l'industrie (Chamiço/Burnay des tabacs);

- avec le grand commerce (Chamiço/Association Commerciale de Lisbonne).

Les privilèges de la BNU sont très importants. Dans la discussion aï a eu lieu à la Chambre des Députés, le député Antonio de Serpa Pimentel lui-même favorable au projet, observait avec quelque réserve qu'en permettant à la BNU de multiples activités on laissait le champ libre à une "promiscuité" d'opérations:

"Je vois dans ce projet, en ce qui concerne l'Outremer, une promiscuité d'opérations que, en tant que règle générale, j'entends qu'on ne doit pas admettre à une Banque de circulation. Des opérations en même temps de circulation, de crédit personnel ou de crédit immobilier, ce sont des attributions que j'entends que le même établissement ne doit pas avoir." 9

Ces privilèges ont donné lieu à une violente polémique dans la presse, surtout dans le Jornal do Comércio: Avec de telles concessions le commerce de la Métropole avec la colonie reste entièrement sous l'emprise, l'arbitre et la préférence de la Banque", écrivait Casimiro da Silva Marques ${ }^{10}$. Quelques jours plus tard, dans le même journal, José Barbosa Leão protestait aussi: "Cette institution qui devrait donner vie aux Provinces d'Outremer, peut, avec un tel statut devenir uniquement une opération de spéculation des actionnaires de la Banque, surtout des plus influents, sans des avantages, plutôt avec des inconvénients pour ces mêmes Provinces"11.

Ce journal observait par ailleurs que, contrairement à la règle habituelle des Banques Coloniales (réserve d'or équivalente à $1 / 3$ de l'émission fiduciaire) les

\footnotetext{
8 Neves Ferreira, ancien Ministre de la Marine et d'Outremer, fut nommé en 1900 Administrateur de la Compagnie Agricole de Cazengo, importante propriété accaparée par la BNU dans une des régions les plus riches de l'Angola.

9 Braga Paixão, 100 anos do Banco Nacional Ultramarino na vida portuguesa (1864-1964) (Lisbonne, 1964) I: 45.

10 lbid., 79.
} 
Statuts étaient rédigés de telle façon que rien ne garantissait que ce principe fondamental serait respecté par la BNU. Si la BNU "n'est pas obligée d'avoir du métal dans les colonies pour payer au porteur les billets que la Banque y créera, si en plus des énormes faveurs qu'elle reçoit, elle a le droit de fabriquer en papier tout l'argent avec lequel elle doit négocier dans les Provinces d'Outremer, alors cette Banque est une chose inqualifiable; et il faut s'étonner qu'il y ait eu un Ministre pour la proposer et un Parlement pour la légaliser"12. Comme nous verrons plus loin ce journaliste ne se trompait guère.

En dépit de toutes les polémiques et de quelques scandales, la succursale de la Banque Nationale d'Outremer a Luanda a commence son activité en 1865, suivie par les agences de St. Tomé, Benguela et Moçâmedes.

III

Le pratique de la BNU en Angola et à St. Tomé

Pour le Ministre Mendes Leal la BNU était une nécessité impérieuse: Donner une forte poussée à l'agriculture, aider puissamment la colonisation, ce n'est possible qu'avec l'effectif concours de capital. Mais pour qu'il soit vraiment fécond il faut que ce capital stimule l'industrie."13

Toutefois, dès le début de son activité à Luanda, la Banque ne paraît pas se conformer exactement à ces objectifs de "développement. " Déjà dans son Rapport de la deuxième année d'exercice (1866) on peut lire que "le taux d'intérêt minimum (...) dans les opérations de cette Banque est de 9 pour cent," alors que le contrat avec le Gouvernement limitait le taux d'intérêt aune maximum de $8 \%^{14}$. On peut même lire dans un travail bien documenté sur la BNU publié en 1893:

"Dans les mains de tous les individus qui ont eu besoin de recourir à la Banque, on trouve des documents prouvant que cette Banque n'a fait aucun cas de cette obligation légale."15

\footnotetext{
11 lbid., 86.

12 Ibid., 86.

13 Ibid., 17.

14 BNU, Relatórios do Banco Nacional Ultramarino desde o ano da sua fundação em 1865 até 1889 (Lisbonne, 1890) 33.

15 Anonyme, Os privilégios do Banco Ultramarino (Lisbonne, 1893), 18.
} 
Un autre aspect fondamental: la quasi totalité des opérations de la BNU sont à court terme, ce qui n'était certainement pas la meilleure manière d'aider l'agriculture comme l'avait voulu le Ministre Mendes Leal.

En effet, la succursale de Luanda reconnaissait en 1867 que les prêts directs à l'agriculture étaient en très petit nombre, "étant donné que beaucoup d'agriculteurs ont utilisé le capital de la Banque par l'intervention du corps commercial de Luanda"16. II s'agissait, en d'autres termes, de prêts faits à de grandes maisons commerciales lesquelles étaient, en même temps, propriétaires de fermes dans le littoral et à l'intérieur, dans l'arrière pays (fazendas). En un mot: c'était un crédit extrêmement sélectif et, pour ainsi dire, au compte-gouttes. Par ailleurs, tout indique qu'il obéissait souvent a un autre critère non avoué; La possibilité de la BNU de venir un jour à prendre possession de ces propriétés en cas de non paiement des intérêts ou de défaut à l'échéance des remboursements.

Si pendant les deux premières années le nombre d'effets de commerce escomptés a doublé (de 109 effets escomptés en 1866 à 266 effets en 1867), peu sont les effets à plus de trois mois et aucun à plus de 6 mois, en dépit du fait que les opérations commerciales sur la place de Luanda étaient, en général, à plus de 6 mois. Cependant quelques firmes "choisies" (sic) recevaient un traitement de faveur sans que l'on parvienne à trouver dans cette pratique de la Banque un critère objectif et uniforme. On lit seulement: "Comme les ventes exigent des délais supérieurs à 6 mois, nous avons consenti à des renouvellements des échéances des effets des bonnes firmes, variant le taux d'intérêt de 9 à $12 \%$ et I'amortissement selon la confiance qu'elles méritent pour nous". ${ }^{17}$

On voit par ces premiers éléments que la pratique de la Banque ne paraît pas correspondre aux propos de ses défenseurs. L'image de "moteur du développement" laisse rapidement place à une autre: celle d'un financier spéculateur contre les intérêts de larges secteurs du capital local lui-même, surtout du petit capital.

Ainsi, dès 1867, la Banque commence à s'emparer des propriétés urbaines de Luanda, premier signe annonciateur de sa future politique. Elle exécute des hypothèques solidement garanties alors que les remboursements n'étaient parfois en retard que de quelques mois, ce qui donne une idée de la rapidité avec laquelle

16 BNU, Relatórios, 53.

17 Ibid., 58-59. 
la Banque sévissait et exemplifie bien la manière don't elle envisageait le "développement" au service duquel elle était supposée travailler.

D’ailleurs la conception "théorique" de la BNU ressort dans ce passage de son Rapport annuel de 1875:

"Les banques - dont on ne peut pas dire qu'elles augmentent le capital d'un pays - distribuent et appliquent le capital existant, facilitant ainsi à l'industrie et à l'économie une augmentation de ce capital. On commet donc une erreur quand on entreprend des actions bancaires, industrielles ou commerciales qui dépassent les forces du capital disponible."18

Il est certain que les prêts sur hypothèques de bonnes propriétés sont une forme sûre et pratiquement sans risques - toutes choses étant égales par ailleurs - de placer les capitaux de la Banque. Mais il serait toutefois difficile de prétendre que c'était là la meilleure manière d'aider au "développement" économique de la colonie. Suivons quelques passages de la vie de la Banque:

En 1881 "la Banque, qui a acquis depuis peu la ferme ("roça") Macambrará, en paiement d'un crédit important, vient de la vendre avec le sacrifice de seulement quelques intérêts du capital. ${ }^{19}$

En 1884 le gérant de la succursale de Luanda a fait une visite d'inspection aux propriétés agricoles hypothéquées à la Banque dans les régions de Quanza, Cazengo et Gulongo Alto, les plus riches régions de café, desservies par le fleuve Quanza et par où passait le projet du Chemin de Fer dont la construction, déjà prévue, commencerait deux ans plus tard précisément.

En 1886 la BNU prendra possession définitive de la plantation de Agua Izé (dans l'lle de St. Tomé), une des plus grandes propriétés de l'archipel de St. Tomé et Principe où travaillaient environ 250 travailleurs "serviçais" (travailleurs supposés être en régime de contrat, mais se trouvant, en réalité, dans une situation de semiesclavage légal, sinon même en esclavage de fait). Cette plantation aura quelques années plus tard, sous l'orientation de la Banque, plus que doublé le nombre de ses travailleurs "serviçais".

En 1887 la succursale de Luanda écrit dans son rapport:

"Dans la Province de l'Angola nous avons été forces d'acquérir la propriété de la plantation Prototypo, dans la région de Cazengo. Le café reçu de cette

\footnotetext{
18 BNU, Relatórios, 60.

19 Ibid., 631.
} 
propriété a atteint cette année 3229 sacs, soit 53276955 reis une fois déduites les dépenses postérieures à l'embarquement dans le port de Luanda."20

Dans le rapport annuel de 1888 on lit à nouveau: "Nous avons été forces d'acquérir par adjudication judiciaire, dans la région de Golungo Alto, la plantation Montalegre et à St. Tomé et à Cap Vert d'autres propriétés rustiques que nous espérons transformer - en leur appliquant le capital indispensable - en sources de rente importante pour la Banque."21

On peu déduire que si les plantations et propriétés rustiques pouvaient être source de rentes lucratives pour la Banque, il est logique qu'elles pourraient l'être également pour ses propriétaires particuliers - si la Banque mettait en pratique les objectifs en fonction desquels elle avait été créée. II n'est pas difficile de voir dans ces spoliations systématiques la philosophie de cette institution financière. Et parce que cela s'avéra une constante de l'activité de la Banque depuis sa fondation jusqu'à son extinction en Angola dans les années 20 de ce siècle, il paraît clair que les critiques et dénonciations dans la presse portugaise de la fin du XIXe siècle, avaient de sérieux fondements.

En 1900 la BNU possédait, rien qu'en Angola "dans les communes de Cazengo et Golungo Alto, six grandes propriétés: Prototypo, Palmira, N'Delagando, Colónia de St. João (...), Cagica et Montalegre."22 La région était maintenant très valorisée par la construction du Chemin de Fer d'Ambaca. Alors la Banque, qui cette fois-ci n'avait voulu vendre aucune de ces propriétés valorisées de jour en jour par le Chemin de Fer, a constitué "avec d'autres capitaux (...) la Compagnie Agricole de Cazengo" qui intégrait toutes les propriétés que l'on vient de citer. Le premier Administrateur de la Compagnie de Cazengo fut Neves Ferreira, ancien Ministre de la Marine et Outremer, maintenant employé par la BNU."23

Dans ces conditions on comprend que les critiques et attaques à la BNU aient continué aussi bien au Portugal qu'en Angola. L'argumentation de la Banque était systématiquement molle et peu convaincante: elle ne mérite donc pas d'être discutée ici. Mais il est par contre intéressant de faire référence aux résultats d'une enquête sur le régime bancaire des colonies effectuée en 1893 par une Commission Parlementaire. Les réponses à cette enquête ne laissent pas

\footnotetext{
20 Ibid., 791.

21 Ibid., 881.

22 Paixão, 100 Anos, 2:275.

23 Ibid.
} 
beaucoup de doutes sur la nature de l'activité de la BNU dans les colonies. Voici, par exemple, da réponse du Gouverneur de I'Angola, Álvaro da Costa Ferreira: "La Banque Nationale d'Outremer ne peut satisfaire les besoins de la Province dans les circonstances actuelles. La Province de I'Angola doit résoudre prioritairement un problème: celui du développement maximum de l'agriculture, compensant ainsi les difficultés du commerce avec les natifs, commerce que les Belges et les Français devient de l'intérieur (de l'Angola) vers l'Etat Indépendant et vers le Congo Français. La BNU ne peut disposer de capitaux en bénéfice de l'agriculture, car elle y a déjà beaucoup employé. Le fort taux d'intérêt des prêts à l'agriculture a compliqué les effets des mauvaises récoltes et des erreurs d'Administration. Tout cela a provoqué l'insolvabilité de la plus grande partie des plantations, d'où leur expropriation, la plus grande partie des plantations de café étant aujourd'hui aux mains de la Banque et d'autres courant le même risque. En ce qui concerne le commerce, la Banque fait aussi beaucoup moins de ce qu'elle pourrait et devrait faire en sa faveur (...). L'industrie n'a reçu aucun concours de la BNU. Encore que l'industrie existante dans la Province soit très faible, même ce petit secteur n'a pas été aidé. Cet état de choses ne doit pas continuer: il est indispensable qu'il y ait une réforme bancaire dans la Province." 24

Les réponses données par le Gouverneur, les fonctionnaires et les commerçants du District du Congo, au Nord de la colonie, furent très semblables. On peut dire aussi que le témoignage apporté par l'Association Commerciale de Luanda, organe représentatif des intérêts du capital local, est révélateur des divergences existantes. En affirmant que la BNU "fausse les fondements et les buts qui ont été à l'origine de sa création", I'Association Commerciale critique violemment la concurrence "ruineuse" faite aux propriétaires locaux par la BNU:

"Ce que la Banque ne paie pas comme contribution industrielle, agricole, impôt de timbre, contribution municipale et tous les autres impôts, représente une économie importante qui lui permet de payer plus cher le rachat de ses serviteurs ("serviçais"), d'acheter plus cher les provisions pour les nourrir, d'augmenter les prix des porteurs qui lui apportent les marchandises aux plantations et ramènent de celles-ci les produits (...). Les autres agriculteurs ne peuvent pas supporter une telle concurrence." 25

\footnotetext{
24 Ibid., 2:217-18 (c'est nous qui soulignons).

25 Ibid., 2:220.
} 
En même temps l'Association Commerciale confirme ce que le Jornal do Comércio affirmait déjà 29 ans auparavant: dans la succursale de Luanda de la BNU, la "réserve obligatoire"(un tiers de l'émission en métal) n'est pas en métal mais en papier:

"La réserve métallique de la Banque, établie dans la loi pour cette succursale, a une forme pratique déplorable (...). Cette réserve est représentée par le papier-monnaie qu'on appelle 'billets du trésor' (cédulas da Junta de Fazenda), lesquels méritent le plus grand crédit local mais ne répondent pas aux besoins du commerce de la Province. Une telle réserve est à l'origine d'un cercle vicieux et, il est triste de le dire, ridicule, car elle place le porteur d'un billet de banque dans la condition de recevoir en échange, dans la Banque, un billet du Trésor, lequel ne peut être échangé au Trésor Public (Fazenda) que par un billet de banque !"26.

En 1901 la polémique autour des privilèges de la BNU augmente parmi les

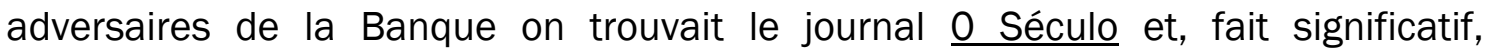
l'Association Commerciale de Porto. La présence de cette dernière dans le camp "opposant" révèle clairement la lutte entre la bourgeoisie mercantile du Nord du pays et l'alliance BNU/bourgeoisie mercantile de Lisbonne pour le contrôle du marché colonial (vins et textiles notamment).

Mais si la BNU est fondamentalement, comme tout porte à croire un organisme financier spéculateur qui ne parait pas valoriser d'une façon tant soit peu significative l'activité économique du secteur colonial de I'Angola et de St. Tomé et Principe, cette Banque montre, en contrepartie, un dynamisme incontestable en faveur de l'accumulation du capital financier métropolitain. Son action couvre plusieurs champs, parfois les plus inespérés pour une institution financière:

- la Banque est un intermédiaire commercial et consignataire?27

- elle embauche et transporte pour les lies de St. Tomé et Principe de la main-d'œuvre africaine ("serviçais" dans des conditions plus que douteuses. Certaines sources parlent même de trafic d'esclaves)

- elle contrôle la circulation mercantile (transports terrestres, fluviaux et maritimes)

\footnotetext{
26 Ibid., 2:222.

27 Un journal publia même un document dans lequel il était prouvé qu'outre un taux d'intérêt très exagéré, le débiteur était obligé de consigner une quantité très importante de café et de cacao sur lesquels la Banque recevait une commission de 3 pour cent que la consignation soit faite ou pas".
} 
- elle canalise vers l'extérieur des capitaux importants (aussi bien vers le Portugal et l'Europe que vers le Brésil)

- elle pratique, dans bien des cas, des taux d'intérêt usuraires ${ }^{28}$.

En tant qu'intermédiaire commercial la Banque, curieusement, pratique même le troc direct transportant des marchandises vers l'intérieur de la colonie et les y échangeant directement, c'est-à-dire, sans l'intervention de monnaie fiduciaire, contre d'autres marchandises qu'elle fait ramener au litoral dans ses barques de la Compagnie de Navigation du fleuve Quanza. Elle reçoit aussi des marchandises à consignation: elle les fait expédier à Lisbonne, les met en stock quand les cotations sont défavorables et exerce les fonctions de véritable intermédiaire commercial (commissions, etc.). De la vente des marchandises la Banque se fait rembourser des prêts octroyés aux agriculteurs, reçoit les intérêts accumulés, les commissions, les frets dans le fleuve Quanza et les frets maritimes dans la liaison LuandaLisbonne, etc.

En 1880 par exemple, la succursale de Luanda prévient le Siège à Lisbonne qu'elle va expédier vers la Métropole entre 1,000 et 1,200 tonnes de café de la dernière récolte, "appartenant à des clients et à quelques autres personnes qui ont besoin d'avances dans cette place"29. Et plus loin:

"Nous avons déjà à Lisbonne, en provenance de Luanda, 900 sacs de café, 166 fardeaux de coton, 125 sacs de caoutchouc, 194 récipients de cire (valant environ 30 millions de réis) (...). A Luanda également, et rien que des propriétaires qui sont nos clients, nous attendons (...) des livraisons supérieures à 1000 tonnes de café valant plus de 200 millions de réis (...). De St. Tomé nous avons aussi à vendre, et par conséquent à porter au crédit de notre Agence de St. Tomé, des marchandises d'un montant de plus de 100 millions de réis, parmi lesquelles 4400 sacs de café, 1800 sacs de cacao."30

Les exemples de ce genre sont innombrables dans les Rapports de la Banque Nationale d'Outremer.

Une autre occupation lucrative et à première vue surprenante, c'était l'embauche de main-d'œuvre africaine pour St. Tomé. Depuis l'installation de son

Cf. Anonyme, Os privilégios, 18.

${ }^{28}$ La même source démontra, chiffres en main, que la Banque pratiqua dans un cas déterminé un taux d'intérêt de $66 \%$ par an. Cf. Ibid.

29 BNU, Relatórios, 579. 
Agence dans cette lie la Banque s'est beaucoup préoccupée du problème de la main-d'œuvre. En faisant la transcription d'un document qu'il prétend avoir reçu de son Agence dans l'île, le siège de la Banque (tout indique que c'est Chamico luimême qui écrit) manifeste d'une façon voilée sa propre opinion sur la question de l'abolition de l'esclavage, ne craignant pas de disserter en matière de doctrine politique:

"Cette mesure (l'abolition) a affecté la propriété de l'agriculteur et, par conséquent, l'intérêt gênerai, mais nous n'avons pas entendu la regretter autant qu'on s'y attendait. Peut-être parce qu'on n'a pas décrété la complète et immédiate liberté de l'esclave, lequel, passant à la condition de libre ("liberto"), continue à être pratiquement un esclave. Mais on proteste, et à juste titre, contre le fait que le gouvernement n'ait pas tenu parole et vienne détruire ce qu'il a promis: payer une indemnisation au propriétaire d'esclaves en 1878. Le gouvernement n'établit pas le travail obligatoire ou un bon règlement du travail indigène (...). On ne doit pas décréter la liberté du Noir (...) sans, en même temps, donner force de loi au travail obligatoire et sans indemniser le propriétaire qui a acquis des droits devent la loi. Cette mesure porte préjudice aussi bien aux intérêts généraux (...) qu'aux intérêts de la Banque, parce que l'agriculture sans bras cesse d'être une activité - et la terre ne vaut plus rien. Quelle garantie pourront offrir les prêts sur de telles hypothèques, ou même sur les biens servant de caution, avec le manque de travailleurs."31

La Banque revient plusieurs fois à la charge sur le problème de la maind'œuvre pour St. Tomé et la nécessité d' "encourager l'émigration" vers cette île. En 1870 la Banque dit même qu'à St. Tome on a perdu un tiers de la récolte de café par manque de bras. Ces événements expliquent "l'anxiété avec laquelle les propriétaires attendent la résolution de la question du travail."32

En 1875 la BNU annonce pour la première fois qu'elle va elle-même embaucher directement de la main-d'œuvre pour St. Tomé. C'est Chamiço lui même qui écrit à ce propos: "On a chargé Mr. Blandy d'embaucher au Libéria et dans d'autres points de la côte 600 hommes et femmes. Le Conseil d'Administration de la Banque a ordonné qu'ils soient remis à son Agence (de St.Tomé) pour être envoyés à quelques unes des principales plantations."33

\footnotetext{
30 Ibid., 598.

31 Ibid., 129.

32 Ibid., 288.

33 Ibid., 368.
} 
Les choses semblent s'être passées à la satisfaction de la BNU et de son Gouverneur, puisqu'à la fin de 1876 Chamiço signalait qu'on avait "importé environ 3000 travailleurs de la côte du Libéria", résultat considéré "positif" même si ce nombre n'était pas encore suffisant selon la Banque.

On se demandera pourquoi la BNU n'allait pas chercher cette main-d'œuvre en Angola au lieu de faire de tels efforts sur la côte occidentale africaine, dans des régions parfois relativement mal connues. La Banque elle-même reconnaissait que la meilleure solution aurait été d'aller chercher ces travailleurs en Angola, "mais on craignait des problèmes avec les vaisseaux de guerre anglais" (lesquels faisaient à cette époque la chasse au trafic d'esclaves au large de la côte africaine, surveillant particulièrement I'Angola). D'ailleurs l'année suivante, le navire à voile Ovarense affrète par un certain Moraes au service de la BNU fut arraisonné et, semble-til, confisqué "par l'autorité anglaise sous prétexte qu'il faisait du commerce d'esclaves." 34

Les protestations renouvelées de la BNU contre le manque de main-d'œuvre (à l'état de semi-esclavage du moins, comme c'était la règle) ont une explication: la Banque s'était emparée de plusieurs riches plantations et était, évidemment, intéressée à leur rentabilité.

En Angola sa politique a été quelque peu différente. Quand les propriétaires ne pouvaient pas rembourser leurs emprunts à la Banque, celle-ci effectuait des saisies comme nous l'avons vu. Mais ce n'était pas l'agriculture qui l'intéressait en premier lieu; c'était la spéculation sur des terres valorisées par la pénétration du Chemin de Fer d'Ambaca, zone où, précisément, se trouvaient les plantations dont la Banque s'était emparée.

En 1877 la BNU fait, pour la première fois dans ses Rapports, référence à 1"exportation" vers St. Tomé de la main-d'œuvre africaine en provenance de Novo Redondo (Angola). L'année suivante la Banque a "signé contrat" avec environ 1800 travailleurs de Novo Redondo et assura leur transport maritime vers St. Tomé dans des conditions épouvantables comme tout semble l'indiquer35.

Cette activité de la Banque a soulevé de nombreuses critiques aussi bien dans la Métropole qu'en Angola. Dans les milieux libéraux, bien entendu, mais aussi parmi les propriétaires de la bourgeoisie coloniale angolaise directement atteints

\footnotetext{
34 lbid., 463.

35 lbid., 487.
} 
par la concurrence de la Banque dont l'action provoquait la rareté de la maind'œuvre.

En 1877 le journal $\underline{0 \text { Progresso }}$ (Lisbonne) publie une correspondance de Luanda ou I'on peut lire:

"En ce moment personne n'ignore à Luanda au'a bord du navire à vapeur Benguela (...) se trouvent 240 esclaves achetés par la Banque Nationale d'Outremer a destination de St. Tomé (...) . Le transport de fruits (sic) vers cette île a pris une expansion étonnante depuis que la BNU a pris possession et administre la plantation Agua Izé (...). On dit que la BNU a dans Novo Redondo 1000 esclaves prêts à partir au premier signe. Le navire Pensamento a lui aussi été affrété pour le transport de 300 ou 400 Noirs pour le compte de la même institution bancaire et avec la même destination."36

De tout ce qui a été dit on peut conclure que la BNU, en dépit des objectifs précis pour lesquels elle avait été créée, a orienté son action au bénéfice exclusif d'une fraction de la bourgeoisie bancaire et financière de la Métropole et au détriment, non seulement des sociétés africaines ("exportation" d'éléments de sa population, par exemple), mais aussi de la bourgeoisie coloniale elle-même, surtout de la petite et moyenne bourgeoisie, que la Banque avait pour "mission" implicite de favoriser en priorité.

Si dans les années 20 de ce siècle la BNU finit par être pratiquement expulsée de l'Angola, cela n'a pas été dû à une gestion défectueuse ou à une distribution excessive et sans critère de crédits, comme certains auteurs le prétendent encore aujourd'hui37. En 1930 Cunha Leal, qui fut Gouverneur de la Banque de l'Angola qui avait pris la succession de la BNU en Angola, affirmait:

"La formation de la plupart, sinon de la totalité, des capitaux actuellement appliqués en Angola, n'a pas été obtenue avec l'emploi de capitaux préexistants mais avec du travail humain accumulé".38 L'affirmation, au contraire de ce qui pourrait paraître à première vue, n'avait aucune intention marxisante. Cunha Leal voulait seulement dire que la contribution du capital bancaire pour le développement de la colonie avait été pratiquement nulle.

\footnotetext{
36 Cf. O Progresso (Lisbonne) du 25/10/1877. Cite par Paixão, 100 anos, 1: 500.

37 "Le crédit, élément de la vie économique, est difficulté dans les Provinces d'Outremer où il n'y a pas de concurrence à cause de l'existence des privilèges et des exclusifs de la BNU." Cf. Miguel de Bulhões, A Fazenda Pública de Portugal (Lisbonne, 1884), 151.

38 Cf. Cunha Leal, Subsídios para $£$ estudo do problema do crédito em Angola (Lisbonne, 1930), 19.
} 
L'inflation par le crédit dont la Banque s'est rendu "coupable", selon beaucoup d'auteurs, dans les années de la décade de 1920, n'a pas obéi à une quelconque volonté de favoriser une politique de "développement", mais fut plutôt une manoeuvre spéculative destinée à jouer sur la dévalorisation de la monnaie angolaise (1'"escudo de l'Angola") par rapport à la livre sterling, selon un texte récemment publie et pendant longtemps inédit de Norton de Matons."39

Déjà en 1927 le Haut-Comissaire de I'Angola, Vicente Ferreira, avait présenté des éléments statistiques qui démontraient le caractère purement spéculateur de la BNU."40

En résumé, si l'on juge l'activité de la Banque Nationale d'Outremer même d'un point de vue libéral, on doit reconnaître qu'après un demi-siècle d'exclusivité et de privilèges exceptionnels, sa pratique ne s'est guère distinguée de celle d'une économie de pillage. Mais ce qui rend son action particulière, c'est moins son insertion, après tout "normale" dans le système classique du Pacte Colonial, que son orientation presque entièrement tournée vers les intérêts de la bourgeoisie mercantile de Lisbonne, au détriment des colons eux-mêmes et, par conséquent, en manifeste préjudice de la consolidation à long ou moyen terme d'une politique colonisatrice avec un minimum de cohérence.

On peut donc prétendre que la BNU fut une institution inadéquate qui, loin de favoriser la pénétration du capitalisme, présenta un caractère quasiment "féodal." En dernière analyse, la BNU fut un obstacle et une des causes du retard de la colonisation portugaise à l'égard des colonisations française ou anglaise, retard qu'elle ne rattrapera plus jamais.

39 Cf. A. Duraes, Angola e o General Norton de Matos, (Lisbonne, 1976) 35.

40 Cf. Vicente Ferreira, "O sistema Monetário de Angola" Estudos Ultramarinos 1(1953), $235 \mathrm{ff}$. 